\title{
Resident microbial load, toxigenic potential and possible quality control measures of six imported seasoning powders on the Ghanaian market
}

\begin{abstract}
Assessment of resident microbiological contamination of six imported seasoning powders on the Ghanaian market were studied using three media DRBC, OGYE and PCA. The presence of moulds and yeasts was compared on two media (DRBC and OGYE) at $30^{\circ} \mathrm{C}$ for 7 days and bacteria on PCA at $35^{\circ} \mathrm{C}$ for $48 \mathrm{hr}$. Determination of elemental compositions and mycotoxin analyses (aflatoxins and ochratoxins) were also analysed using AAS spectrometry and HPLC technique respectively. As a means of eliminating resident microbial load in the seasonings, gamma irradiation doses of $0,2,5$, and $10 \mathrm{KGy}$ were applied at an absorbed dose rate of $1.683 \mathrm{KGy} / \mathrm{hr}$. seven genera of fungi (Aspergillus, Cladosporium, Penicillium, Absidia, Eurotium, Rhizopus and Paecilomyces) were isolated from the samples. Aspergillus species (A. candidus, A. flavus, A. niger, A. oryzae, A. sulphureus) predominated over the other species followed by Penicillium (P. corylophilum, P. aurantiogriseum); Cladosporium (C. herbarum, C. macrocarpum) and Rhizopus (R. oryzae, R. stolonifer). The percentage occurrence profile of resident fungi changed after 6months storage. Species of bacteria encountered varied from one seasoning to the other but included Acinetobacter pittii, Bacillus funiculatus, B. subtilis, Filifactor villosus, Lactobacillus equi, L. malferment, $L$. quasseri and Pseudomonas orientalis. Lactobacillus species predominated over the other bacteria genera encountered. All the seasonings contained variable high to low contents of $\mathrm{Fe}, \mathrm{Na}, \mathrm{K}, \mathrm{Ca}$ and $\mathrm{Mg}$. The heavy metals occurred in low or trace amounts namely $\mathrm{Cu}, \mathrm{Zn}, \mathrm{Pb}, \mathrm{Cr}, \mathrm{Ni}, \mathrm{Co}, \mathrm{Ar}$ and $\mathrm{Hg}$. Benny shrimp powder contained high concentration of $\mathrm{K}(4240 \mathrm{mg} / \mathrm{l})$ and $\mathrm{Na}(424.20 \mathrm{mg} / \mathrm{l})$, while Onga chicken seasoning contained high $\mathrm{Na}$ $(13,700 \mathrm{mg} / \mathrm{l})$; Remie chicken seasoning powder contained $104.82 \mathrm{mg} / \mathrm{l}$ of Fe. Aflatoxins (B1, B2, G1 and G2) and ochratoxin A were not detected in all the test samples but there was the presence of Monosodium Glutamate. Gamma irradiation dose of 5-10KGy decontaminated the samples.
\end{abstract}

Keywords: seasoning powders, bacterial, fungal, aflatoxins, ochratoxin a, monosodium glutamate, elemental composition
Volume 8 Issue I - 2018

\begin{abstract}
Odamtten GT,' Nartey LK,' Wiafe Kwagyan M,'Anyebuno G, ${ }^{2}$ Kyei Baffour $V^{2}$

'Department of Plant and Environmental Biology, University of Ghana, Ghana

${ }^{2}$ Council for Scientific and Industrial Research, Food Research Institute, Ghana
\end{abstract}

\begin{abstract}
Correspondence: Wiafe Kwagyan M, College of Basic \& Applied Sciences, Department of Plant and Environmental Biology, University of Ghana, P. O. Box LG 55 Legon, Accra, Ghana,Email mwiafe-kwagyan@ug.edu.gh, wiakwa@yahoo.com
\end{abstract}

Received: November 14, 2017 | Published: January 19, 2018
Abbreviations: GMP, good manufacture process; HAACP, hazard analysis of the produce at the critical control point; ICMSF, international commission on microbiological specification for food; RTC, radiation technology centre; AAS, atomic absorption spectrometry; HPLC, high pressure liquid chromatography; ANOVA, analysis of variance; ISO, international standards organization; WHO, world health organization

\section{Introduction}

Seasonings are substances such as salt, pepper, spice or herb added as flavour to food. These seasonings also improve aroma, taste, colour and pungency. They can be used in the fresh state, dried, powdered or mixed ingredients. Seasoning powders are mixtures of seasoned flavouring components such as salt, sugar, spices etc. hence spices can be considered as a sub-set of seasoning. ${ }^{1}$

Most of the raw materials used in preparing seasoning powders are cultivated in warm and humid tropics and some in temperate climates where a variety of fungal and bacterial contaminants are resident in stored raw material. The post-harvest handling operations of gathering ingredients for manufacture of seasoning powders begin in the field level of picking raw materials with their attendant field microflora, drying to acceptable moisture content levels (i.e. sundrying, oven drying or open) subject to the vicissitude of the weather). Other basic operations such as harvesting, transporting, threshing, drying, cleaning packaging and storage processing of seasoning are potential sources of contamination with pathogens which can lead to an increased number of food-borne infections and intoxications. ${ }^{2-4}$

If, Good Manufacture Process (GMP) and Hazard Analysis of the Produce at the Critical Control Point (HAACP) is presumed to have been carried out, the sterilization/pasteurization process should keep the product stable in quality within the stipulated expiry date. However, opening the packaging may breach the integrity of the ideal conditions for extension of shelf-life and so even under refrigeration; the product may be predisposed to contamination. The liberalized economy in developing countries such as Ghana and impact of the media advertisement has resulted in the influx of many goods especially food products in addition to our locally manufactured ones such as Maggi onion cubes, Maggi Shrimp cube, Royco Beef cube, 
Royco Shrimp cube etc. to mentioned but a few. ${ }^{4}$ Some of the recent brands in the Ghanaian market are Benny Beef stock, Benny Crayfish powder stock, Remie Chicken seasoning powder, Napa Valley Jollof rice seasoning, Onga seasoning powder and Indomie Seasoning powder.

In Ghana, assessment of microbiological contamination on some selected spices have been reported by Caulley; Painstil; Addo; Appenteng, Ahene, Odamtten and Owusu, Yankey to mention but few. These researchers recorded the occurrence of different fungal and bacterial species in fresh and powdered dehydrated samples. They recorded the following fungal species Aspergillus flavus, A. fumigatus, A. nidulans, A. alutaceus, A. glaucus, A. niger, Cladosporium herbarum, Fusarium sp., Penicillium digitatum and Trichoderma viride. These species showed five patterns of infection $A$. niger, A. fumigatus and $A$. nidulans displayed a continuous decline in population during the threemonths storage period. On the one hand, some bacterial species recorded included Enterobacter cloacae, E. amnigenus, E. sakazakii, Flavobacterium sp., Chromobacterium violaceum, Pesudomonas putida, P. aeruginosa, P. cepacia, Acinetobacter sp. and Serratia plymuthica, Aeromonas salmonicida and Pseudomonas. ${ }^{4,5}$

In Costa Rica, the microbiological quality of some powdered spices usually used in homes, without further thermal treatment was evaluated. Black and white and green pepper counts gave plate counts and faecal coliforms counts exceeded the International Commission on Microbiological Specification for Food (ICMSF) standards and that contamination was probably due to drying conditions and postharvest treatment Arce (as cited in Ahene \& Odamtten et al. ${ }^{4}$

These constituted a serious health hazard to the consumer of such spices on the market. Fungi associated with spices, herbs, codiments, cereals and dehydrated products are predominated by Aspergillus species majority of which strains produce mycotoxins including the potent aflatoxins Flannin and Hui. ${ }^{4}$ These mycotoxigenic species impart toxic metabolites during growth in food and food products making food unfit for human consumption. The mycotoxins with the most potential for human hazard are toxins of storage fungi in the genera Aspergillus, Penicillium and Fusarium. The most potent are aflatoxins B1, B2, G1 and G2 (produced by Aspergillus flavus, A. parasiticus, A. nomius). Fumonisin (Fusarium verticilloides), trichothecene (Fusarium spp); Patulin (P. expansum, Paecilomyces sp.). ${ }^{4}$

Odamtten ${ }^{6}$ reiterated that the danger of mycotoxicoses especially aflatoxicoses still exists in Africa and cannot be discounted in our health delivery system. However, in some instances despite the presence of these mycotoxigenic species in the raw spices and spice products there is self-protection against mycotoxin products by virtue of the formulation and inherent preservation offering self-protection. For example, Ahene \& Odamtten et al., ${ }^{4}$ showed that aniseed, rosemary, royco and maggi cubes offered self-protection against aflatoxin formation by $A$. flavus by creating condition unfavourable for toxin formation. Benny Beef powdered stock, Benny Crayfish powdered stock, Remie Chicken seasoning powder, Napa Valley Jollof Rice seasoning, Onga Chicken seasoning and Indomie seasoning are popular imported seasoning powders on the shelf of local supermarkets and shops in Ghana. Their formulation although stated on the packaging material is not known for certain by the consuming public and cannot be taken for granted. This paper report our findings on work carried out to assess the microbiological quality of these listed seasonings and provides information on the aflatoxigenic potential of the resident
Aspergillus flavus and A. alutaceus isolated from these products and the possible control measures via gamma irradiation energy.

\section{Materials and methods}

The packaged seasoning powders selected for the study have the following composition according to the manufacturers and specific flavour enhancers stated in this study are numbered in E numbering system are listed below:

E102: Tartrazine; E110: Sunset Yellow FCF; Orange Yellow S; E121: Orcein, Orchil; E122: Azorubine, Carmoisine; E124: Ponceau 4R, Cochineal Red A; E133: Brilliant Blue FCF; E330: Citric acid; E363: Succinic acid; E551: Silicon dioxide; E621: Monosodium L-glutamate; E627: Disodium guanylate; E631: Disodium inosinate

\section{Materials}

Benny beef powdered stock: Ionised salt, cornflour, flavour enhancers (monosodium glutamate MSG E621, E631, E627, flavouring anticaking agent (E551), colourant E122, E133, E124, Tartrazine (E102, E110).

Benny crayfish powdered stock: Ionised salt, monosodium glutamate MSG E621, cornflour, disodium ionosinate, crayfish powder, approved colourant, E121, flavouring herbs, spices.

Remie chicken seasoning powder: Ionised salt, monosodium glutamate MSG E621, corn starch, sugar, vegetable fat, natural colours, spices, chicken seasoning powder.

Napa valley jollof rice seasoning: Ionised salt, monosodium glutamate MSG E621, starch, sugar, maltodextrin, tomato powder.

Onga chicken seasoning: Iodated salt, maize, flavour enhancers (E621, E631, E627), maltodextrin, sugar, anticaking agent (E551), spice extracts (turmeric, capsicum, chilli) vegetable fat, flavourants, acids (E363, E330), herbs.

Indomie seasoning: Ionised salt, flavour enhancers (E621, E631, E627) sugar, garlic powder, yeast extract, dry leek, onion powder, chicken flavour, spices.

\section{Methods}

a) Microbiological enumeration: This was done according to the method described by ISO. ${ }^{7}$ Fungi and bacteria in the seasoning powders were determined as prescribed using the decimal serial dilution technique up to $1: 10^{3}$. $1 \mathrm{ml}$ aliquots of the serially diluted samples were placed in either $20 \mathrm{ml}$ of Dichloran Rose Bengal Chloramphenicol agar (DRBC, CM 727) or Oxytetracycline Glucose Yeast Extract agar (OGYE, Oxoid 545, for fungal count or on Plate Count Agar, PCA (Biochemika 70152 (for bacterial count). Plates containing PCA were incubated at $35^{\circ} \mathrm{C}$ for $48 \mathrm{hrs}$ which plates containing DRBC and OGYE were incubated at $28-30^{\circ} \mathrm{C}$ for 7 days. At the end of the prescribed incubation periods bacterial and fungal counts obtained were calculated as $\log _{10} \mathrm{CFU} / \mathrm{g}$ sample.

b) Identification of fungi encountered: Fungi isolated were identified by their cultural, colour morphological characteristics (microscopic and macroscopic) according to the standard reference of Samson \& Barnett et al. ${ }^{8,9}$

c) Identification of bacterial species: This was carried out using the novel Matrix-Assisted Laser desorption/ionization time of flight (MALDI-TOP-MS) Mass Spectrometry method prescribed by the 
manufacturer. ${ }^{10}$ It is an analytical method of identification and is characterised by the fast and precise assessment of mass of molecules in a variable range of $100 \mathrm{Da}$ to $100 \mathrm{KDa}$. About $0.1 \mu \mathrm{l}$ of Bacterial Test Standard (BTS) of Escherichia coli with $\mathrm{HCl}$ was added and allowed to dry. The prepared target plates were put in the Maldi Biotype Galaxy (Microflex) chamber according to the manufacturer's instructions using a scanner. The codes of the target plates were scanned and the names of the bacteria appeared automatically on the computer.

d) Influence of radiation treatment on the survival of resident fungi and bacteria in sample: The samples were placed in duplicate in envelopes and sent to the Radiation Technology Centre (RTC) of the Biotechnology and Nuclear Agriculture Institute of the Ghana Atomic Energy Commission Kwabenya, Accra for gamma irradiation treatment at $0,2,5$ and $10 \mathrm{KGy}$. Irradiation was carried out in air at dose rate of $1.683 \mathrm{KGy} / \mathrm{h}$ (Gamma Conveyor Irradiator, Budapest, Hungry). The absorbed doses were ascertained by Fricke Dosimetry measured spectrophotometrically at $303 \mathrm{~nm}$ wavelength in the peak of the absorption spectra.

e) After irradiation the decimal serial dilution technique up to $1: 10$ was used to estimate surviving population and the incubation temperature and media, fungi and bacteria are described above. Surviving fungi and bacteria were identified as spelled out above.

f) Determination of elemental composition of samples: The following elements were analysed for their presence and concentration [iron $(\mathrm{Fe})$, sodium $(\mathrm{Na})$, potassium $(\mathrm{K})$, calcium $(\mathrm{Ca})$, magnesium $(\mathrm{Mg})$, copper $(\mathrm{Cu})$, zinc $(\mathrm{Zn})$, lead $(\mathrm{Pb})$, chromium $(\mathrm{Cr})$, nickel (Ni), cobalt (Co), mercury ( $\mathrm{Hg}$ ) and arsenic (As)] using an Atomic Absorption Spectrometry AAS technique (Model 240FS, Vienna, Austria) following the instructions prescribed by the manufacturer.

g) Mycotoxin analyses of samples: The aflatoxins (by Aspergillus flavus) and Ochratoxin A (by A. alutaceus) levels in the samples were determined using the High Pressure Liquid Chromatography HPLC (Model Agilest 1260 Series, USA) at the Food Research Institute CSIR, Ghana following the methods outlined by Pons. ${ }^{11}$ The samples were treated differently; one batch was the unadulterated seasonings incubated at $85 \%$ ERH for $72 \mathrm{~h}$ to induce fungal growth (control) and the other batch of the same samples were spiked with $2.5 \times 10^{3}$ spores $/ \mu$ of either A. flavus or A. alutaceus and stored at $85 \% \mathrm{ERH}$ for $72 \mathrm{~h}$ before analysis for mycotoxins.

h) Statistical analysis: The results, where appropriate were analysed statistically using Stat-graphics plus Analysis of Variance (ANOVA) to evaluate data at $\mathrm{p}<0.05$ level of significance. Differences between means were determined using Least Significant Test LSD.

\section{Results}

\section{A. Microbiological quality of the six dehydrated seasoning powders}

The population of fungi in the six dehydrates powders varied depending on the medium used in the isolation. The fungal population varied from a low $0.20 \log _{10} \mathrm{CFU} / \mathrm{g}$ to $2.54 \log _{10} \mathrm{CFU} / \mathrm{g}$ sample (Table1). Generally, Onga chicken seasoning and Benny Beef stock seasoning powder harboured more fungal population. The population of fungi resident in the samples isolated on OGYE was higher by $0.25-1.36 \log _{10}$ cycles than on DRBC.

Total aerobic bacterial population varied from $0.50 \log _{10} \mathrm{CFU} / \mathrm{g}$ sample (Indomie Seasoning Powder) to $1.40 \log _{10} \mathrm{CFU} / \mathrm{g}$ sample (Napa Jollof Seasoning Powder).
Table I Microbiological quality of the indicated seasoning products plated on either DRBC or OGYE (for fungi) and PCA (for bacteria)

\begin{tabular}{|c|c|c|c|}
\hline \multirow{2}{*}{ Type of Seasoning } & \multicolumn{3}{|c|}{ Total microbial population $\left(\log _{10} \mathrm{CFU} / \mathrm{g}\right)$ on } \\
\hline & DRBC (Fungi) & OGYE (Fungi) & $\begin{array}{l}\text { PCA } \\
\text { (Bacteria) }\end{array}$ \\
\hline Benny Beef Stock & $\mathrm{I} .48$ & 2.39 & 1.3 \\
\hline Remie Chicken & 0.2 & 0.4 & 1.3 \\
\hline Napa Valley Jollof & $\mathrm{I} .48$ & 2 & $\mathrm{I} .4$ \\
\hline Onga Chicken & 1.18 & 2.54 & 0.2 \\
\hline Benny Srimp Stock & 1.3 & 1.54 & I \\
\hline $\begin{array}{l}\text { Indomie Powder } \\
\text { Seasoning }\end{array}$ & 1.18 & $\mathrm{I} .48$ & 0.5 \\
\hline
\end{tabular}

In all the seasoning powders seven genera of fungi (Aspergillus, Cladosporium, Penicillium, Eurotium, Rhizopu and Paecilomyces) were isolated from the two media. Aspergillus species (A. candidus, A. flavus, A. niger, A. oryzae, A. sulphureus) predominated over the species encountered followed by Penicillium ( $P$. corylophilum, $P$. aurantiogriseum $=P$. verrucosum var cylopium and Cladosporium (C. herbarum, C. macrocarpum) and Rhizopus (R. oryzae, $R$. stolonifer). Three other genera encountered were Absidia, Eurotium, and Paecilomyces with single species (Table 2). The percentage occurrence profile of the resident fungal species changed after 6months storage (Table 3). For example, occurrence of $A$. flavus in Benny shrimp stock powder changed from $14.3 \%$ to nil in 6 months; in Napa Jollof Seasoning, it changed from initially none to $22.2 \%$ in 6months; in Onga chicken seasoning percentage occurrence of $A$. flavus changed from none initially to $12.6 \%$ in 6 months.

sulphureus population in Benny Shrimp Stock seasoning changed from an initial $14.3 \%$ to nil in 6 months (Table 3 ); Indomie seasoning powdered harboured $77.8 \%$ of $P$. aurantiogriseum but reduced to $36.2 \%$ in 6months. Population of the same fungus in Onga chicken seasoning powder changed from initial of $23.1 \%-46.2 \%$ in 6 months; in Benny Shrimp Stock, population declined from $35.7 \%$ to $26.1 \%$ in 6months (Table 3).

Bacterial species isolated from the six samples initially and after 6months storage are presented in Table 4. About 3-7 bacterial species were resident initially in all the seasoning samples. But this changed to nil to seven in 6 months depending on the type of sample Table 4 .

The species of bacteria isolated were Acinetobacter pittii, Bacillus funiculatus, B. subtilis, Filifactor villosus, Lactobacillus equi, L. malferment, L. quasseri and Pseudomonas orientalis. Lactobacillus species predominated over the genera encountered (Table 4).

\section{B. Influence of gamma irradiation on the microfloral population}

Increasing irradiation doses from $0-10 \mathrm{KGy}$ proportionately decreased the fungal and bacterial population in all the samples. A dose of 5-KGy eliminated the bacterial floral while it left a residual of Rhizopus oryzae mycoflora in all the samples (Table 5) (Table 6).

\section{Elemental composition of the seasoning powders}

All the seasonings were high in iron content (3.6-104.82mg/l), 
Sodium (43.4-13700mg/l), Potassium (0.5-4240mg/1), Calcium (0.088-2.105mg/l), and Magnesium (0.035-0.37mg/l).

The remaining heavy metals occurred in low or trace amounts namely Copper, Zinc, Lead, Chromium, Nickel, Cobalt, Arsenic and Mercury) (Table 7). Benny shrimp powder contained high concentration of K $(4240 \mathrm{mg} / 1)$ and sodium $(424.0 \mathrm{mg} / 1)$, while Onga chicken seasoning contained high amount of sodium $(13780 \mathrm{mg} / \mathrm{l})$. On the other hand, Remi chicken seasoning powder had high iron content (104.82mg/l) (Table 7).

D. Determination of total aflatoxin and ochratoxin A content of seasoning samples

The fresh seasoning samples were stored at $85 \%$ ERH for $72 \mathrm{~h}$ to induce fungal growth and those spiked with spores of A. flavus and kept the same way did not contain aflatoxins B1, B2, G1 and G2 in all the test samples (Table 8). The same was true for Ochratoxin A which was not detected in any other samples (Table 9).

Table 2 Total list of mycoflora isolated from the six seasoning powders at $30^{\circ} \mathrm{C}$ on two different media DRBC and OGYE

\begin{tabular}{|c|c|}
\hline S. no & Mycoflora \\
\hline I & Absidia Glauca Hagem ${ }^{5}$ \\
\hline 2 & Aspergillus Candidus Link ${ }^{3}$ \\
\hline 3 & A. Flavus Link $k^{3-5}$ \\
\hline 4 & A.Alutaceus Wilhelm' \\
\hline 5 & A. Oryzae Cohn ${ }^{3}$ \\
\hline 6 & Za. Niger Van Tieghem $1,3,4$ \\
\hline 7 & A. Sulphureus (Fres) Thom And Church ${ }^{1-3,5}$ \\
\hline 8 & A. Terreus Thom' \\
\hline 9 & Cladosporium Herbarium (Pers) Link ${ }^{4-6}$ \\
\hline 10 & C. Macrocarpum Preuss' \\
\hline II & Eurotium Amstelodanii Mangin ${ }^{5}$ \\
\hline 12 & Penicillium Corylophilum Dierckx ${ }^{4,5}$ \\
\hline 13 & P.Aurantiogriseum Dierckx (P.Verrucosum Var Cyclopium) $)^{4,6}$ \\
\hline 14 & Paecilomyces Carneus (Duché Et Heim) Brown \& Smith² \\
\hline 15 & Rhizopus Oryzae Went \& Prinsen Geerlings ${ }^{1-5}$ \\
\hline 16 & R. Stolonifer Link ${ }^{4}$ \\
\hline
\end{tabular}

Key LKI, benny beef stock seasoning; LK2, remie chicken seasoning; LK3, napa valley jollof seasoning; LK4, onga chicken seasoning; LK5, benny shrimp stock seasoning; LK6, indomie seasoning

Table 3 Percentage occurrence of the indicated fungal species resident in the fresh samples and those stored for 6 months at $30^{\circ} \mathrm{C}$ beyond the expiry date

\begin{tabular}{|c|c|c|c|c|c|c|c|c|c|c|c|c|}
\hline \multirow{3}{*}{ Fungus isolated } & \multicolumn{12}{|c|}{ Type of seasoning and percentage occurrence (\%) } \\
\hline & \multicolumn{2}{|l|}{ LK I } & \multicolumn{2}{|c|}{ LK 2} & \multicolumn{2}{|c|}{ LK 3} & \multicolumn{2}{|l|}{ LK 4} & \multicolumn{2}{|l|}{ LK 5} & \multicolumn{2}{|c|}{ LK 6} \\
\hline & $\mathbf{F}$ & 6mth & $\mathbf{F}$ & 6mth & $\mathbf{F}$ & 6mth & $\mathbf{F}$ & 6mth & $\mathbf{F}$ & 6mth & $\mathbf{F}$ & 6mth \\
\hline Aspergillus flavus & - & - & - & - & - & - & - & - & 14.3 & 17.5 & - & - \\
\hline Aspergillus niger & 54.5 & 31.4 & - & 29 & 42.9 & 33.5 & 30.8 & 53.8 & - & 56.5 & - & - \\
\hline Aspergillus sulphureus & - & - & - & 45.2 & 35.7 & 15.7 & - & - & 14.3 & - & - & - \\
\hline Cladosporium herbarum & - & 20 & - & - & - & - & 30.8 & - & - & - & 22.2 & 12.3 \\
\hline Cladosporium macrocarpum & 31.8 & - & - & - & - & - & - & - & - & - & - & - \\
\hline $\begin{array}{l}\text { P. Aurantiogriseum }=(p . \text { Verrucosum } \\
\text { var. Cyclopium) }\end{array}$ & - & 34.3 & - & 25.8 & - & 13.7 & 23.1 & 46.2 & 35.7 & 26.1 & 77.8 & 36.2 \\
\hline Rhizopus oryzae & 13.6 & 14.3 & 100 & - & 21.4 & - & 7.7 & - & 7.1 & - & - & - \\
\hline Rhizopus stolonifer & - & - & - & - & - & - & 7.7 & - & - & - & - & - \\
\hline
\end{tabular}

Key F, fresh samples (Initial); LK I, benny beef stock seasoning; LK 2, remie chicken seasoning; LK 3, napa valley jollof seasoning; LK 4, onga chicken; seasoning; LK 5, benny shrimp stock seasoning; LK 6, indomie seasoning 
Table 4 List of Bacterial species isolated from fresh samples initially and the sample stored for 6 months and plated on plate count agar at $35^{\circ} \mathrm{C}$ for 48 hours

\begin{tabular}{|c|c|c|c|c|c|c|c|c|c|c|c|c|}
\hline \multirow{3}{*}{ Bacterial species } & \multicolumn{12}{|c|}{ Sample code and period of storage in Month } \\
\hline & \multicolumn{2}{|c|}{ LK I } & \multicolumn{2}{|c|}{ LK 2} & \multicolumn{2}{|c|}{ LK 3} & \multicolumn{2}{|c|}{ LK 4} & \multicolumn{2}{|c|}{ LK 5} & \multicolumn{2}{|c|}{ LK 6} \\
\hline & 0 & 6mth & 0 & 6mth & 0 & 6mth & 0 & 6mth & 0 & 6mth & 0 & 6mth \\
\hline Acinetobacter pitii & + & + & - & - & - & - & - & - & - & - & - & - \\
\hline Bacillus funiculatus & - & - & + & + & - & - & - & - & - & - & - & - \\
\hline Bacillus subtilis & - & + & + & + & - & - & - & + & + & + & + & - \\
\hline Filifactor villosus & + & + & + & + & - & - & - & - & - & - & - & - \\
\hline Lactobacillus equi & + & + & + & + & + & - & + & + & + & + & + & - \\
\hline Lactobacillus malferment & + & + & + & + & + & - & - & - & - & - & - & - \\
\hline Lactobacillus quasseri & + & + & + & + & + & - & - & - & + & - & + & - \\
\hline Pseudomonas orientalis & - & - & + & + & - & - & - & + & - & + & - & - \\
\hline
\end{tabular}

Key LK I - LK 6; +: Present; -:Absent

Table 5 Radiation response of the indicated bacteria species resident in the six seasoning powders

\begin{tabular}{|c|c|c|c|c|c|}
\hline \multirow{2}{*}{ Types of seasoning } & \multicolumn{5}{|c|}{ Bacterial species surviving and dose applied (Kgy) } \\
\hline & & 0 & 2 & 5 & 10 \\
\hline \multirow{7}{*}{ LK I (Benny Beef Stock) } & Acinetobacter pitii & + & - & - & - \\
\hline & B. subtilis & - & - & - & - \\
\hline & B. funiculatus & + & - & - & - \\
\hline & F. villosus & - & - & - & - \\
\hline & L. equi & + & + & - & - \\
\hline & L. malferment & + & - & - & - \\
\hline & L. quasseri & + & - & - & - \\
\hline \multirow{8}{*}{ LK 2 (Remie Chicken Powder) } & A. pitii & + & - & - & - \\
\hline & B. subtilis & + & - & - & - \\
\hline & B. funiculus & + & - & - & - \\
\hline & F. villosus & + & - & - & - \\
\hline & L. equi & + & - & - & - \\
\hline & L. malferment & + & - & - & - \\
\hline & L. quasseri & + & - & - & - \\
\hline & P. orintalis & + & - & - & - \\
\hline \multirow{4}{*}{ LK 3 (Jollof Seasoning) } & L. equi & + & + & + & - \\
\hline & L. malferment & + & - & - & - \\
\hline & L. quasseri & + & - & - & - \\
\hline & P. orintalis & - & - & - & - \\
\hline \multirow{2}{*}{ LK 4 (Onga Chicken Seasoning) } & L. equi & + & + & - & - \\
\hline & L. quasseri & - & - & - & - \\
\hline
\end{tabular}


Table continued..

\begin{tabular}{|c|c|c|c|c|c|}
\hline \multirow[t]{2}{*}{ Types of seasoning } & \multicolumn{5}{|c|}{ Bacterial species surviving and dose applied (Kgy) } \\
\hline & Bacillus subtilis & + & - & - & - \\
\hline \multirow[t]{3}{*}{ LK 5 (Benny Shrimp Stock) } & L. equi & + & - & - & - \\
\hline & L. quasseri & + & + & - & - \\
\hline & B. subtilis & + & - & - & - \\
\hline \multirow{3}{*}{ LK 6 (Indomie Seasoning) } & L. equi & + & - & - & - \\
\hline & L. quasseri & + & + & - & - \\
\hline & F. villosus & + & - & - & - \\
\hline
\end{tabular}

Table 6 Radiation response of the indicated fungal species resident in the six seasoning powders

\begin{tabular}{|c|c|c|c|c|c|}
\hline \multirow{2}{*}{ Type of seasoning } & \multicolumn{5}{|c|}{ Fungal species surviving and the applied dose (KGy) } \\
\hline & & 0 & 2 & 5 & 10 \\
\hline \multirow{11}{*}{ LK I (Benny Beef Stock) } & Aspergillus alutaceus & + & - & - & - \\
\hline & A. oryzae & + & - & - & - \\
\hline & A. sulphureus & + & - & - & - \\
\hline & A. terreus & + & - & - & - \\
\hline & Cladosporium herbarum & + & - & - & - \\
\hline & Fusarium solani & + & - & - & \\
\hline & F. verticillioides & + & - & - & - \\
\hline & Penicillium digitatum & + & - & - & - \\
\hline & P. aurantiogriseum $=(P$. verrucosum var. cyclopium $)$ & + & + & - & - \\
\hline & Rhizopus oryzae & + & - & - & + \\
\hline & R. stolonifer & + & - & - & - \\
\hline \multirow{6}{*}{ LK 2 (Remie Chicken Seasoning) } & Aspergillus flavus & + & - & - & - \\
\hline & A. niger & + & + & - & - \\
\hline & A. sulphureus & + & - & - & - \\
\hline & C. herbarum & + & - & - & - \\
\hline & P. aurantiogriseum $=(P$. verrucosum var. cyclopium & + & - & - & - \\
\hline & R. oryzae & + & - & - & + \\
\hline \multirow{9}{*}{ LK 3 (Napa Valley Jollof Seasoning) } & Aspergillus niger & + & - & - & - \\
\hline & A. oryzae & + & - & - & - \\
\hline & A. sulphureus & + & - & - & - \\
\hline & Cladosporium herbarum & + & - & - & - \\
\hline & P. aurantiogriseum $=(P$. verrucosum var cyclopium & + & - & - & - \\
\hline & P. corylophilum & + & + & - & - \\
\hline & R. oryzae & + & - & - & + \\
\hline & R. stolonifera & + & - & - & - \\
\hline & Syncephalastrum racemosum & + & - & - & - \\
\hline
\end{tabular}


Table continued...

\begin{tabular}{|c|c|}
\hline & 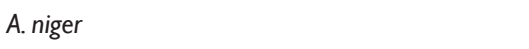 \\
\hline & A. sulphureus \\
\hline & C. herbarum \\
\hline LK 4 (Onga Chicken Seasoning) & P. aurantiogriseum $=(P$. verrucosum var cyclopium \\
\hline & R. oryzae \\
\hline & R. stolonifer \\
\hline & Aspergillus niger \\
\hline & A. sulphureus \\
\hline & Cladosporium herbarum \\
\hline & Fusarium solani \\
\hline LK 5 (Benny Shrimp Stock Powder) & P. corylophilum \\
\hline & P. aurantiogriseum $=(P$. verrucosum var. cyclopium \\
\hline & R. oryzae \\
\hline & R. stolonifer \\
\hline & A. niger \\
\hline & A. oryzae \\
\hline & A. suphureus \\
\hline 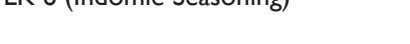 & C. herbarum \\
\hline & P. aurantiogriseum $=(P$. verrucosum var cyclopium \\
\hline & R. oryzae \\
\hline
\end{tabular}

Table 7 Sample analysis for elemental composition of the seasoning powders

\begin{tabular}{|c|c|c|c|c|c|c|}
\hline \multirow{3}{*}{$\begin{array}{l}\text { Elemental } \\
\text { conc. }(\mathrm{mg} / \mathrm{l})\end{array}$} & \multicolumn{6}{|c|}{ Types of sample: Spices } \\
\hline & LKI & LK2 & LK3 & LK4 & LK5 & \multirow{2}{*}{$\begin{array}{l}\text { Instrument } \\
\text { detection limit } \\
(\mathrm{mg} / \mathrm{l})\end{array}$} \\
\hline & $\begin{array}{l}\text { Powdered } \\
\text { beef stock }\end{array}$ & $\begin{array}{l}\text { Benny } \\
\text { powdered } \\
\text { stock }\end{array}$ & $\begin{array}{l}\text { Napa valley joll of } \\
\text { rice seasoning }\end{array}$ & $\begin{array}{l}\text { Onga } \\
\text { chicken }\end{array}$ & $\begin{array}{l}\text { Remie chicken } \\
\text { seasoning }\end{array}$ & \\
\hline Iron & 7.23 & 6.48 & 3.6 & 9.72 & 104.8 & 0.006 \\
\hline Manganese & $<0.002$ & $<0.002$ & $<0.002$ & $<0.002$ & $<0.002$ & 0.002 \\
\hline Coper & $<0.003$ & $<0.003$ & $<0.003$ & $<0.003$ & $<0.003$ & 0.003 \\
\hline Zinc & $<0.001$ & 1.26 & 0.96 & 0.54 & 1.2 & 0.001 \\
\hline Calcium & 0.677 & 0.689 & 0.088 & 2.105 & 0.463 & 0.001 \\
\hline Magnesium & 0.27 & 0.271 & 0.035 & 0.837 & 0.812 & 0.0003 \\
\hline Lead & $<0.001$ & $<0.001$ & $<0.001$ & $<0.001$ & $<0.001$ & 0.001 \\
\hline Chromium & 1.02 & 0.1 & 0.84 & 2.88 & I.148 & 0.001 \\
\hline Nickel & $<0.001$ & $<0.001$ & $<0.001$ & $<0.001$ & $<0.001$ & 0.001 \\
\hline Cadmium & 0.906 & $<0.002$ & $<0.002$ & $<1.020$ & $<0.002$ & 0.002 \\
\hline Sodium & 43.4 & 424 & 59.5 & 13700 & 43.8 & 0.01 \\
\hline Cobalt & 1.14 & $<0.005$ & $<0.005$ & $<0.005$ & $<0.005$ & 0.005 \\
\hline Potassium & 14 & 4240 & 0.5 & 9.1 & 4.7 & 0.01 \\
\hline Arsenic & $<0.001$ & $<0.001$ & $<0.001$ & $<0.001$ & $<0.001$ & 0.01 \\
\hline Mercury & $<0.001$ & $<0.001$ & $<0.001$ & $<0.001$ & $<0.001$ & 0.01 \\
\hline
\end{tabular}


Table 8 Aflatoxin analysis of seasoning samples with the indicated codes with or without Aspergillus flavus inoculation after 5 days at $30^{\circ} \mathrm{C}$ using $\mathrm{HPLC}$ technique

\begin{tabular}{|c|c|c|c|c|c|}
\hline \multirow{2}{*}{ Sample Code } & \multicolumn{5}{|c|}{ Aflatoxin $(\mu \mathrm{g} / \mathrm{Kg})$} \\
\hline & B I & B2 & G I & G2 & Total \\
\hline LK I (FRI MTR:Af) & ND & ND & ND & ND & ND \\
\hline LK 2 ( FRI MTR:Af) & ND & ND & ND & ND & ND \\
\hline LK 3 ( FRI MTR:Af) & ND & ND & ND & ND & ND \\
\hline LK 4 (FRI MTR:Af) & ND & ND & ND & ND & ND \\
\hline LK 5 (FRI MTR:Af) & ND & ND & ND & ND & ND \\
\hline LK6 (FRI MTR:Af) & ND & ND & ND & ND & ND \\
\hline LK I (FRI MTR: C) & ND & ND & ND & ND & ND \\
\hline LK 2 (FRI MTR: C) & ND & ND & ND & ND & ND \\
\hline LK 3 (FRI MTR: C) & ND & ND & ND & ND & ND \\
\hline LK 4 (FRI MTR: C) & ND & ND & ND & ND & ND \\
\hline LK 5 (FRI MTR: C) & ND & ND & ND & ND & ND \\
\hline LK 6 (FRI MTR: C) & ND & ND & ND & ND & ND \\
\hline
\end{tabular}

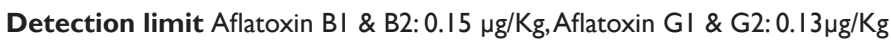

LK I, benny beef stock seasoning; LK 2, remie chicken seasoning; LK 3, napa valley jollof seasoning; LK 4, onga chicken seasoning; LK 5, benny shrimp stock seasoning; LK 6, indomie seasoning; (FRI MTR:Af) Samples spiked with $2.5 \times 10^{3}$ CFU/g sample of Aspergillus flavus;(FRI MTR: C) Control raw sample without Aspergillus flavus

Table 9 Ochratoxin analysis of seasoning samples with the indicated codes with or without Aspergillus alutaceus after 5 days at $30^{\circ} \mathrm{C}$ using $\mathrm{HPLC}$ technique

\begin{tabular}{ll}
\hline Sample Code & Ochratoxin A $(\mu \mathrm{g} / \mathbf{k g})$ \\
\hline LK I (FRI MTR:Aa) & ND \\
LK 2 ( FRI MTR:Aa) & ND \\
LK 3 (FRI MTR:Aa) & ND \\
LK 4 (FRI MTR:Aa) & ND \\
LK 5 (FRI MTR:Aa) & ND \\
LK6 (FRI MTR:Aa) & ND \\
LK I (FRI MTR: C) & ND \\
LK 2 (FRI MTR:C) & ND \\
& \\
LK 3 (FRI MTR: C) & \\
&
\end{tabular}

\section{Detection limit Ochratoxin, $0.83 \mu g / \mathrm{Kg}$}

LK I -LK 6, benny beef stock seasoning; LK 2, remie chicken seasoning; lk 3, napa valley jollof seasoning; LK 4, onga chicken seasoning; LK 5, benny shrimp stock seasoning; LK 6, indomie seasoning;(FRI MTR:Aa) Samples spiked with 2.5xI03 CFU/g sample of Aspergillus alutaceus; (FRI MTR: C) Control raw sample without Aspergillus alutaceus 


\section{Discussion}

The liberalized global economy has resulted in the influx of many good as well as shoddy food products into developing countries. The agencies responsible for ascertaining the quality of those products from the exporting countries are presumed to have done due diligence to adherence of food quality standards of the International Standards Organisation. However, the survival of microbiological species in dehydrated products in a well-known phenomenon.

Although the population of resident mycoflora with low $(0.20$ $2.54 \log _{10} \mathrm{CFU} / \mathrm{g}$ sample) there were species of pathological importance resident in all the six samples (Table 2) such species included (Aspergillus flavus, A. alutaceus, A. candidus, A. niger, A, sulphureus, Penicillium aurantiogriseum and Paecilomyces). The preponderance of Aspergillus and Penicillium species in dehydrated foods in storage is a well-known phenomenon in the tropics and elsewhere and has been well-documented in Ghana. ${ }^{4,11,12}$ In this paper potential toxinproducing fungal species such as Aspergillus candidus (kojic acid), A. flavus (aflatoxins B1, B2, G1 and G2), A. oryzae (kojic acaid), A. sulphureus (ochratoxins, sterigmatocystin), Cladosprium herbarum (epicladosporic acid), Eurotium amstelodami (sterigmatocystin), Penicillium corylophilum (griseofulvin, roquefortine C), $P$. aurantiogriseum (penicillic acid, viridicatin, ochratoxin, citrinin), Paecilomyces (patulin) have been isolated (Table 2). ${ }^{14}$

The health implications of consuming such contaminated seasonings are enormous in Africa for the consumer who may use an opened sachet bought for use over a long period even beyond the expiry date. The high occurrence of A. flavus, A. niger, A. sulphureus, $P$. corylophilum, $P$. aurantiogriseum in the sample (6.7-77.8\%) kept for 6 months leave much to be desired. The seasonings were also very hygroscopic and deliquescent. For example Indomie seasoning absorbed moisture fast as even 55\%ERH, (data not shown). At all the ERH's studied (55, 75, 85, $95 \mathrm{ERH})$, it took 15-20days for equilibrium to be achieved with the surrounding (Data not shown). This implies that the seasonings were not manufactured to withstand the vicissitude of the changing fortunes of the tropical weather conditions and find purpose in being dissolved quickly in the product earmarked for seasoning. In the event of improper sealing of the package after opening, the moisture content value may increase giving the microorganism the opportunity to form and multiply even if kept under refrigeration.

There has been a correlation between Hepatitis B and aflatoxins in Africa Peers et al. ${ }^{4}$ Liver incidence varied over a 5-fold range, and was strongly associated with estimated levels of aflatoxins Peers et al. ${ }^{4}$

Aflatoxins produced by A. flavus, A. parasiticus, and A. nomius in various food commodities including, maize, rice, barley, wheat, sorghum, groundnut and oil foods, copra, cocoa beans and some spices and codiments have attracted the attention of a number of international health authorities including WHO. ${ }^{15}$ Aflatoxins are immunosuppressive, mutagenic, teratogenic and carcinogenic in their effect with the main target organ being the liver. Evaluation of epidemiological and laboratory results by International Agency for Research in Cancer IARC ${ }^{4}$ showed that there is sufficient evidence in humans for carcinogenicity of naturally occurring aflatoxins. Consequently, aflatoxins are classified as Group1 carcinogens. Acute aflatoxins in humans has been reported in Kenya ${ }^{15}$ Mozambique, Swaziland, Transkei (Van Resenberg \& Schakwyk; Makaula). ${ }^{4,6}$
Ochratoxins produced by A. alutaceus are nephrotoxins of human health importance. Co-occurrence of aflatoxin B1, Fumonisin B, Ochratoxin A and Zearalenone in cereals, dehydrated products and groundnut have been reported. ${ }^{16}$ In Ghana, Wareing et al., ${ }^{12}$ detected sterigmatocystin, patulin, cyclopiazonic acid, penicillic acid, tenuazonic acid, aflatoxins in kokonte (cassava flour) while Kpodo et al., ${ }^{12}$ found the co-occurrence of high levels of aflatoxins, ochratoxins and citrinin in fermented maize dough and fumonisins and their cooccurrence with aflatoxin in maize.

However, in our present study analysis for aflatoxins B1, B2, G1, G2 and ochratoxin A in the control and spiked samples of seasonings showed that both mycotoxins were not formed Tables $8 \& 9$. Presumably, the seasonings products were stable and their formulation, offered self-protection against aflatoxin and ochratoxin. A formation by $A$. flavus and A. alutaceus respectively. This agrees with the findings of Ahene \& Odamtten et al. ${ }^{4}$ Four of the seasonings (Benny Beef powder stock, Benny Crayfish powder stock, Remie chicken seasoning powder, Napa Valley Jollof rice seasoning) contained unspecified amount of monosodium glutamate, MGS, in addition to unspecified ionized salt and flavor enhancers and garlic. These additives could have acted in concert to prevent mycotoxin formation. However, MSG is the sodium salt on the non-essential amino acid glutamic acid. Glutamic acid is the most abundant amino acids in human food. When glutamate is present in free form, not as a component of proteins or peptides, it has a flavour-enhancing effect and for this reason it is added to foods as it is purified monosodium salt FSANZ, ${ }^{17}$ Win,${ }^{18}$ Adeyemo OA. ${ }^{19}$

Levels of free glutamate in foods of animal origin are generally low (beef $33 \mathrm{mg} / 100 \mathrm{~g}$; cow's milk $2 \mathrm{mg} / 100 \mathrm{~g}$ ). Higher levels are found in vegetables $(30-200 \mathrm{mg} / 100 \mathrm{~g})$ and in seasonings, sauces and restaurant meals. ${ }^{17,20}$ The debate on the safety of MSG in food consumed in the developing countries and elsewhere is the range of adverse reaction observed including the symptom complex known as Chinese restaurant syndrome (CRS) and bronchoconstriction in some asthmatic individuals. This syndrome is characterized by headaches, numbness/tingling, flushing, muscle lightness and generalized weakness. ${ }^{21}$ The average person in the USA consumes $0.5-1.0 \mathrm{~g}$ of MSG in a day, ${ }^{22}$ comparable to an average daily dietary exposure of $0.6 /$ day of MSG in the UK with extreme consumers having exposure of more than $2 \mathrm{~g} /$ day e.g. in Taiwan. ${ }^{22}$

Estimated average daily dietary MSG exposure in developing countries has been reported in the range $0.3-1.0 \mathrm{~g} /$ day. ${ }^{23}$ While research indicate that people differ widely in their sensitivity to MSG; ${ }^{22}$ many claim harmful effect due to it. ${ }^{18,24}$ It is up to national safety regulation agencies to ban or prescribe the safety limit of MSG in food although in this present paper it could have played a role in the prevention of mycotoxin formation in the six seasonings. The other interesting observation was that bacterial species isolated from the six seasoning samples varied from an initial 3-7 to none to 7 in 6 months (Table 4) depending on the type of seasoning.

Generally, the bacteria species isolated were Acinetobacter pittii, Bacillus funiculatus, B. subtilis, Filifactor vilbosus, Lactobacillus equi, L. malferment, L. quasseri and Pseudomonas orientalis. Lactobacilli predominated over the other bacteria encountered. The bacterial species listed are being recorded for the first time in seasonings currently on sale in Ghana and many constitute a health hazard. 
Infections associated with Acinetobacter species include ventilator - associated pneumonia, skin and soft-tissue infections, wound infections, urinary tract infections, peritonitis, secondary meningitis and blood stream infections. ${ }^{25}$ Such infections are caused predominantly by members of the A. baumanni complex; infections caused by other species belonging to the genus Acinetobacter are relatively unusual and are restricted mainly to catheter-related blood stream infections and rare outbreaks related to point-source contamination. ${ }^{25}$ Acinetobacter may also cause purulent meningitis. ${ }^{26}$

Lactobacilli on the other hand are part of the normal flora of the human mouth gastrointestinal tract and female genital tract ${ }^{26,27}$ and are generally regarded as safe for use in food (probiotic and to ferment food). ${ }^{27}$ Lactobacillus infections are rare; but can occur opportunistically, especially in immunocompromised individuals. ${ }^{26}$ Most deaths associated with Lactobacillus infections are in individual with underlying immunosuppressive conditions.

Lactobacillus has been found present in the mouth implicated in dental caries, intestines and typically in the adult vagina. ${ }^{26,28}$ On the other hand, L. maleferment has been identified as having low pathogenic potential reinforcing the fact that it is a safe microbe. ${ }^{29}$ L. quasseri infection is accompanied by severe inflammation of the intestinal mucosal surface. Strains of $L$. quasseri produced active bacteriocins Gassericin A which is bactericidal without causing lysis. ${ }^{26}$

Bacillus subtilis is generally non- pathogenic but has occasionally caused foodborne gastroenteritis leading to food poisoning. B. subtilis is an endospore forming bacterium and the endospore it forms enables it to withstand extreme temperatures as well as dry environments. ${ }^{26}$ This same bacterium has also been associated with septicemia, meningitis, endocarditis, pneumonia, wound infections, and other suppurative lesions, particularly as an opportunistic pathogen. ${ }^{26}$

Pseudomonas can be found in many different environments such as soil, water, plant and animal tissue. Many different species of this bacterium are opportunistic pathogens which affect humans, animals and plants. For example, $P$. aeruginosa almost never infects uncompromised tissue but can practically infect any tissue which has some types of compromised defenses. ${ }^{26}$ The presence of bacteria causing diarrhoea, gastrointestinal infection and meningitis etc in the seasoning kept for a long time leave much to be desired as these diseases account for an annual mortality rate of five million people worldwide. ${ }^{30}$ Presence of pathogenic bacteria and potential mycotoxic fungi is not desirable for product stability and safety for human consumption.

All the six seasoning powders showed varied amount of Fe content (3.6-104mg/l), Na (43.4-13700mg/l) K (0.5-4240mg/l) (Table 7). The $\mathrm{WHO}^{31}$ limit for iron intake is $15 \mathrm{mg} / \mathrm{kg}$. Iron deficiency (anaemia) affects one third of the world's population but excessive intake in iron is associated with the risk of colorectal cancer. ${ }^{32}$ The presence of high potassium $(4240 \mathrm{mg} / \mathrm{l})$ over sodium $(424.0 \mathrm{mg} / \mathrm{l})$ was found in Benny Shrimp powder. The presence of high content of potassium over sodium in diet suggests effectiveness against hypertension. Indeed Patil \& Wiafe-Kwagyan et al. ${ }^{33,34}$ also reported a balance between high potassium and low sodium in oyster mushrooms (Pleurotus ostreatus and P. eous) on different substrates. Manzie \& Mattila et al. ${ }^{35,36}$ also found high potassium and low sodium concentrations in the fruiting bodies of mushrooms. Onga chicken seasoning, on the contrary, contained very low potassium $(9.1 \mathrm{mg} / \mathrm{l})$ and very high sodium content (13700mg/l) (Table 7) and may be having the opposite effect of predisposing consumers to hypertension.
The remaining samples contained low traces of $\mathrm{Cu}, \mathrm{Zn}, \mathrm{Ca}, \mathrm{Mg}$, $\mathrm{Pb}, \mathrm{Co}, \mathrm{Ni}, \mathrm{Ar}, \mathrm{Hg}$ (Table 7). Heavy metals such as $\mathrm{Cu}, \mathrm{Mn}, \mathrm{Pb}, \mathrm{Zn}, \mathrm{Ni}$, $\mathrm{Co}, \mathrm{Hg}$ were also detected albeit in small concentrations. Some heavy metals $\mathrm{Fe}, \mathrm{Cu}, \mathrm{Mn}, \mathrm{Zn}$, are essential since they play an important role in the biological systems. Lead is not essential and can be toxic in trace amounts. ${ }^{37}$ However, the essential heavy metals can be toxic when is excessive intake (such as being added to soups and stews as ingredients of seasonings). ${ }^{38,39}$ Five heavy metals were detected in the sporophore (fruiting body) of $P$. eous and $P$. ostreatus $(\mathrm{Cu}, \mathrm{Fe}, \mathrm{Mn}, \mathrm{Pb}$, $\mathrm{Zn})$ by Wiafe-Kwagyan ${ }^{34}$ in trace quantities lower than the reported permissible limit set by WHO. ${ }^{31}$

Copper is an essential constituent of metallo-enzyme and is required in haemoglobin synthesis and in catalysis of metabolic growth. ${ }^{40}$ Lead is toxic even in trace levels. ${ }^{41}$ The impairment of human functions related to lead toxicity includes abnormal size and haemoglobin content of erythrocytes, hyper stimulation of erythropoiesis and inhibition of haemosynthesis Vonugopal \& Wiafe-Kwagyan. ${ }^{34}$ It is therefore of extreme health significance that $\mathrm{Pb}$ was detected in all the samples of the seasonings albeit in trace amounts. However, these values were far below the $10 \mathrm{mg} / \mathrm{kg}$ limit set by $\mathrm{WHO}^{31}$ for $\mathrm{Pb}$ in plant materials. In the event of continuous consumption of foods and soups prepared with such seasonings containing $\mathrm{Pb}$, the cumulative effect on the essential organs of consumers cannot be discounted or ruled out. Manganese is essential metal as it plays an important role in biological systems such as its presence in metallo proteins. ${ }^{37}$ The level of $<0.002 \mathrm{mg} / 1$ detected in the seasonings would not pose immediate danger as this value falls below the toxicity limit of $1000 \mathrm{mg} / \mathrm{kg}$ in plants prescribed by WHO. ${ }^{31}$ Interestingly, varying ranges of $\mathrm{Mn}$ content in mushroom have been documented as $12.9-93.3 \mathrm{mg} / \mathrm{kg}^{42}$ and $14.2-67.7 \mathrm{mg} / \mathrm{kg}^{43} 0.116-0.86 \mathrm{mg} / \mathrm{kg} .{ }^{34}$

Zinc is also an essential mineral which is a component of wide range of enzymes and co-enzymes. It performs catalytic, structural and regulating roles in biosynthesis. According to Ma \& Belt, ${ }^{34}$ zinc contributes to about $33 \mathrm{ppm}$ to adult weight, and is also a constituent of many enzymes involved in the physiological functions of protein synthesis and enzyme metabolism. The WHO recommended limit for zinc in foods in $60 \mathrm{mg} / \mathrm{kg}$. The amount detected in the seasonings varies from $<0.001-1.26 \mathrm{mg} / \mathrm{l}$ far below their permissible level.

\section{Conclusion}

In the concluding experiment of this paper, the possible control of the resident mycoflora and bacterial in the six seasoning was studied using gamma irradiation (Tables 4-6). The WHO/FAO/ IAEA Joint Expert Committee has since 1980 approved the use of gamma irradiation up to $10 \mathrm{KGy}$ for food preservation without any adverse effect on organoleptic and functional properties. ${ }^{44}$ Over 64 countries are currently using this process in diverse ways to prolong the shelf-life of foods for human and livestock consumption. ${ }^{44} \mathrm{In}$ this present study all bacteria species were eliminated by a dose of $5 \mathrm{KGy}$ and above. While $10 \mathrm{KGy}$ of gamma irradiation left a residual, insignificant trace of Rhizopus in the samples (Table 5) (Table 6). It is therefore feasible to use gamma irradiation up to $10 \mathrm{KGy}$ of gamma irradiation to decontaminate the seasoning powders and thus extend the shelf-life of the pre-packaged products and render them safe for human consumption.

It is well-known that both micro and macro-nutrients are required for proper growth of fungi. These mineral elements $\mathrm{P}, \mathrm{K}, \mathrm{Mg}, \mathrm{S}, \mathrm{Na}$ and $\mathrm{Ca}$ are required for growth as sporulation of fungi Wiafe-Kwagyan. ${ }^{34}$ The widely studied micronutrients for the growth of fungal species 
include $\mathrm{Fe}, \mathrm{Zn}, \mathrm{Al}, \mathrm{Mn}, \mathrm{Cu}, \mathrm{Cr}, \mathrm{Mo},{ }^{45-47}$ some of which were detected in the six seasonings used in this present study. The influence of the nutrients in the seasoning on the growth and toxin production of some of the resident predominant fungal species in the seasonings will be reported in a subsequent paper.

\section{Acknowledgements}

We are grateful to the Government of Ghana through the University for partial support of this project.

\section{Conflicts of interest}

Authors declare that there is no conflict of interest.

\section{References}

1. Tainter DR, Grenis AT. Spices and seasonings: A Food Technology Hand book. 2nd ed. Estados Unidos, USA:John Willey \& Sons; 2001. 250p.

2. Buckenhiiskes HJ, Rendlen M. Hygienic problems of phytogenic raw materials for food production with special emphasis to herbs and spices. Food Sci Biotechnol. 2004;13(2):262-268.

3. Alam Khan K, Abrahem M. Effect of irradiation on quality of spices. International Food Research Journal. 2010;17(4):825-836.

4. Ahene RE, Odamtten GT, Owusu E. Fungal and bacterial contaminants of six spices and spice products in Ghana. African Journal of Environmental Science and Technology. 2011;5(9):633-640.

5. Yankey J. Assessment of microbiological contamination of some indigenous spices sold in selected markets in Kumasi metropolis. Kumasi: Kwame Nkrumah University of Science and Technology; 2014.

6. Odamtten GT. Natural Occurrence, Economic Impact and Control of Aflatoxins in Africa. WHO Expert Committee Meeting on Aflatoxins and Health. Brazaville, Republic of Congo, Africa; 2005.

7. International Organisation for Standardisation. Microbiology of food and animal feeding stuff-preparation of test samples, initial suspension and decimal dilutions for microbiological examinations-part I. General rules for preparation of the initial suspension ans decimal dilutions. ISO. 1999:1-6887.

8. Samson RA, Van Reenen-Hoekstra ES. Introduction to food-borne fungi. 3rd ed. Europe: Published by Institute of the Royal Netherlands Academy of Arts and Sciences; 1988.

9. Barnett HL, Hunter BB. Illustrated Genera of Imperfect Fungi. 4th ed. St. Minnesota, USA: APS Press; 2006. 217p.

10. Bruker. Instructions for Bruker Bacteria Test Standard. Bruker Scientific Services, Germany; 2014.

11. Pons WA Jr. High Pressure Liquid Chromatographic Determination of Aflatoxins in Corn. J Assoc Official Anal Chem. 1979;62(3):586-594.

12. Addo AA. Mycological profile and aflatoxigenic potential of resident Aspergillus species of six packed Ghanaian dehydrated foods. Department of Botany, University of Ghana, Legon; 2008. 157p.

13. Appenteng MA. Spoilage fungi of twelve herbal medicines sold in the local markets in Ghana and their aflatoxigenic potential. Department of Botany, University of Ghana, Legon; 2009. 234p.

14. Frisvad JC. Fungal species and their specific production of mycotoxins. In:Samson RA, ES van Reenen-Hoestra, editors. Introduction to FoodBorne Fungi. Central bureau avaoor Schinmeleutures Delft. Institute of the Royal Netherlands Academy of Arts and Sciences the Netherlands, Europe; 1988. p. 239-247.

15. Outbreak of aflatoxin poisoning-Eastern Central Provinces. CDC Centre for Disease Control and Prevention. Kenya; 2004;53(34):790-793.
16. Sangare-Tigoro B, Moukha S, Kouadio HJ, et al. Co-occurrence of aflatoxin B1, fumonisin B1, ochratoxin A and zearalenone in cereals and peanuts from Cote d'Ivoire. Food Addit Contam. 2006;23(10):1000-1007.

17. Monosodium Glutamate. A Safety Assessment. Food Standards Australia New Zealand (FSANZ) Technical Report Series No. 20 Canberra, Australia; 2003.

18. Win DT. MSG-flavour enhancer or deadly killer. AUJT. 2008;12(1):43-49.

19. Adeyemo OA, Farinmade AE. Genotoxic and cytotoxic effects of food flavour enhancer, monosodium glutamate (MSG) using Allium cepa assay. African Journal of Biotechnology. 2013;12(13):1459-1466.

20. Rhodes J, Tiltherly AC, Norman JA, et al. A survey of monosodium glutamate content of foods and an estimation of the dietary intake of monosodium glutamate. Food Addit Contam. 1991;8(5):663-672.

21. Monosodium Glutamate MSG. New Zealand Food Safety Authority (NZFSA). 2017.

22. Hodgson AS. Some facts about Monosodium Glutamate MSG. Food and Nutrition. Cooperative Extension Service. College of Tropical Agriculture and Human Resources CTAHR, University of Hawaii at Manoa; 2001

23. Geha RS, Beiser $\mathrm{A}$, Ren $\mathrm{C}$, et al. Review of alleged reaction to monosodium glutamate and outcome of a multicentre Double-Blind Placebo-Controlled Study. J Nutr. 2000;130(4):10585-10625

24. Tawfik MS, Al-Badr. Adverse effect of monosodium glutamate on liver and kidney function in adult rats and potential protective effect of vitamins C and E. Food and Nutrition Sciences. 2012;3(5):651-659.

25. Peleg AY, Seifert H, Paterson DL. Acinetobacter baumanii:emergence, of a successful pathogen. Clin Microbiol Rev. 2008;21(3):538-582.

26. Ananthanarayan R, Paniker CRJ. Textbook of microbiology. 8th ed. University Press, India Private Limited; 2012. 746p.

27. Cannon JP, Lee T, Bolanos JT, et al. Pathogenic relevance of Lactobacillus, a retrospective review of over 200 cases. Eur J Clin Microbiol Infect Dis. 2005;24(1):31-40.

28. Shivakumar KM, Vidya SK, Chandu GN. Dental caries vaccine. Indian Journal of Dental Research. 2009;20(1):99-106.

29. Lactobacillus fermentum. Wikipedia. 2016.

30. Brooks GF, Butel J, Morse SA. Jawetz, Melnick and Adelberg's Medical Microbiology. 22nd ed. Lange Medical Books/McGraw Hill Medical; 2001. 245p.

31. Evaluation of certain food additives and contaminants. 26th Report of the Joint FAO/WHO Experts Committee on Food Additives. WHO Technical Report Series No 632, Geneva, Switzerland; 1982:35-48.

32. Senesse P, Meance S, Cottet V, et al. High dietary iron and copper and risk of colorectal cancer:a case - control study in Burgundy, France. Nutr Cancer. 2004;49(1):66-71.

33. Patil SS, Ahmed SA, Telang SM, et al. The nutritional value of Pleurotus ostreatus (Jacq: Fr.) Kumm cultivated on different lignocellulosic agrowastes. Innovative Romanian Food Biotechnology. 2010;7:66-76

34. Wiafe-Kwagyan M. Comparative bioconversion of rice lignocellulosic waste and its amendments by two oyster mushrooms (Pleurotus species) and the use of the spent mushroom compost as bio-fertilizer for the cultivation of tomato, pepper and cowpea. Department of Botany, University of Ghana, Legon; 2014.

35. Manzi P, Gambelli L, Marconi S, et al. Nutrients in edible mushrooms:an interspecies comparative study. Food Chemistry. 1999;65(4):477-482. 
36. Mattila P, Könkö K, Eurola M, et al. Contents of vitamins, minera elements, and some phenolic compounds in cultivated mushrooms. $J$ Agric Food Chem. 2001;49(5):2343-2348.

37. Unak P, Lambrecht FY, Biber FZ, et al. Iodine measurements by isotope dilution analysis in drinking water in Western Turkey. $J$ Radioanalytical Nuclear Chem. 2007;273(3):649-651.

38. Al-khlaifat AL, Al Khashman OL. Atmospheric heavy metal pollution in Aquaba City, Jordan using Phoenix dactylifera leaves. Atmos Environ. 2007;41(39):8891-8897.

39. Gopalani M, Shahare M, Ramteke DS, et al. Heavy metal content of potato chips and biscuits from Nagpur City, India. Bull Environ Contam Toxicol. 2007;79(4):384-387.

40. Silvestre MD, Lagarda MJ, Farra R, et al. Copper, iron and zinc determination in human milk using FAAS with microwave digestion. Food Chemistry. 2000;68(1):95-99.

41. Dobaradaren S, Kaddafi K, Nazmara S, et al. Heavy metals (Cd, Cu, Ni and $\mathrm{Pb}$ ) content in fish species of Persian Gulf in Bushehr Port, Iran. $A J$ Biotech. 2010;9(32):6191-6193.
42. Tüzen M. Determination of heavy metals in soil, mushroom and plant samples by atomic absorption spectrometry. Micro Chem J. 2003;74(3):289-297.

43. Soylak M, Saracoglu S, Tuzen M, et al. Determination of trace metals in mushroom samples in Mushroom samples from Kayseri,Turkey. Journal of Food Chemistry. 2005;92(4):649-652.

44. IAEA. Radiation, people and the environment. International Atomic Energy. Agency Vienna, Austria; 2004.

45. Wang D, Sakoda A, Suzuki M. Biological efficiencyand nutritional value of Pleurotus ostreatus cultivated on spent beer grain. Biores Tchnol. 2001;78(3):293-300.

46. Beuchat L, Komitopoulou E, Betts R, et al. Persistence and survival of pathogens in dry foods and dry food processing environments. 2011. $48 \mathrm{p}$.

47. Trienekens J, Zaubier P. Quality and safety standards in the food industry, development and challenges. International Journal of Production Economics. 2008;113(1):107-122. 